This item was submitted to Loughborough's Research Repository by the author.

Items in Figshare are protected by copyright, with all rights reserved, unless otherwise indicated.

\title{
Spallation of thin films driven by pockets of energy concentration
}

\section{PLEASE CITE THE PUBLISHED VERSION}

http://dx.doi.org/10.1016/j.tafmec.2017.04.011

\section{PUBLISHER}

(c) Elsevier Ltd

\section{VERSION}

AM (Accepted Manuscript)

\section{PUBLISHER STATEMENT}

This work is made available according to the conditions of the Creative Commons Attribution-NonCommercialNoDerivatives 4.0 International (CC BY-NC-ND 4.0) licence. Full details of this licence are available at: https://creativecommons.org/licenses/by-nc-nd/4.0/

\section{LICENCE}

CC BY-NC-ND 4.0

\section{REPOSITORY RECORD}

Harvey, Christopher, Bin Wang, and Simon Wang. 2017. "Spallation of Thin Films Driven by Pockets of Energy Concentration”. Loughborough University. https://hdl.handle.net/2134/24864. 


\title{
Spallation of thin films driven by pockets of energy concentration
}

\author{
Christopher M. Harvey ${ }^{\mathrm{a}}$, Bin Wang ${ }^{\mathrm{a}}$ and Simon Wang ${ }^{\mathrm{a}, \mathrm{b}, *}$ \\ ${ }^{a}$ Department of Aeronautical and Automotive Engineering, Loughborough University, \\ Loughborough, Leicestershire LE11 3TU, UK \\ ${ }^{b}$ College of Machinery and Equipment Engineering, Hebei University of Engineering, Handan, China
}

\begin{abstract}
A hypothesis is made that delamination can be driven by pockets of energy concentration (PECs) in the form of pockets of tensile stress and shear stress on and around the interface between a thin film and a thick substrate, where PECs can be caused by thermal, chemical or other processes. Based on this hypothesis, three analytical mechanical models are developed to predict several aspects of the spallation failure of elastic brittle thin films including nucleation, stable and unstable growth, size of spallation and final kinking off. Both straightedged and circular-edged spallations are considered. The three mechanical models are established using partition theories for mixed-mode fracture based on classical plate theory, first-order shear-deformable plate theory and full 2D elasticity. Experimental results show that all three of the models predict the initiation of unstable growth and the size of spallation very well; however, only the 2D elasticity-based model predicts final kinking off well. The energy for the nucleation and stable growth of a separation bubble comes solely from the PEC energy on and around the interface, which is 'consumed' by the bubble as it nucleates and grows. Unstable growth, however, is driven both by PEC energy and by buckling of the separation bubble. Final kinking off is controlled by the fracture toughness of the interface and the film and the maximum energy stored in the separation bubble. This work will be particularly useful for the study of spallation failure in thermal barrier coating material systems.
\end{abstract}

Keywords: buckling; crack propagation and arrest; energy release rate; fracture mechanisms; residual stress

\footnotetext{
* Corresponding Author
}

Email addresses: c . m . harvey@lboro . ac . uk (Christopher M. Harvey), s.wang@lboro.ac.uk (Simon Wang), b.wang2@lboro.ac.uk (Bin Wang) 


\begin{tabular}{|c|c|}
\hline \multicolumn{2}{|c|}{ Nomenclature } \\
\hline$A$ & amplitude of upward deflection of film bubble \\
\hline$b$ & width of through-width straight-edged delamination \\
\hline$h$ & thickness of film \\
\hline$E$ & Young's modulus of film \\
\hline $\bar{E}$ & effective Young's modulus of film \\
\hline$G, G_{I}, G_{I I}$ & total, mode I and mode II ERRs \\
\hline$G_{I c}, G_{I I c}$ & film-substrate pure mode I and II interface fracture toughness \\
\hline$G_{c}$ & film-substrate mode-dependent interface fracture toughness \\
\hline $\bar{G}_{c}$ & film-substrate interface fracture toughness averaged over delamination \\
\hline$G_{c f}$ & fracture toughness of film material \\
\hline$M_{x B}, N_{x B e}$ & crack tip longitudinal bending moment and effective force \\
\hline$M_{r B}, N_{r B e}$ & crack tip radial bending moment and effective force \\
\hline$r, \theta$ & radial and circumferential coordinates of circular film bubble \\
\hline$R_{B}$ & $\begin{array}{l}\text { half-crack length of straight-edged delamination; radius of circular-edged } \\
\text { delamination }\end{array}$ \\
\hline$u_{0}$ & residual strain energy density in the film \\
\hline$U_{a}$ & $\begin{array}{l}\text { 'bubble energy'; increase in combined strain energy and surface energy due } \\
\text { to bubble separation }\end{array}$ \\
\hline$U_{b}$ & bending strain energy of film bubble \\
\hline$U_{i}$ & in-plane strain energy of film bubble \\
\hline$U_{s}$ & surface energy of delaminated surfaces of film bubble \\
\hline$U_{0}$ & strain energy of film bubble before separation \\
\hline$w$ & upward deflection of film bubble \\
\hline$x, y$ & lengthwise and widthwise coordinates of straight-edge film bubble \\
\hline$z$ & out-of-plane coordinate \\
\hline$\alpha$ & buckling correction factor (e.g. due to initial imperfection) \\
\hline$\beta$ & kink-off angle \\
\hline$\varepsilon_{x}^{R}, \sigma_{x}^{R}$ & averaged axial relaxation strain and stress due to bending deflection \\
\hline$\varepsilon_{r}^{R}, \sigma_{r}^{R}$ & averaged radial relaxation strain and stress due to bending deflection \\
\hline$\varepsilon_{0}, \sigma_{0}$ & uniform residual compressive strain and stress in film \\
\hline$v$ & Poisson's ratio of film \\
\hline$\psi$ & ratio of film-substrate pure mode II and I interface fracture toughness \\
\hline
\end{tabular}

\section{Introduction}

Thin solid films are found in many different applications fulfilling various roles [1] such as confinement of electric charge in integrated electronic circuits, thermal insulation in thermal barrier coatings (TBCs), and protection against corrosion, friction and wear in surface coatings. Although thin films are not usually expected to have a primary load- 
carrying capability, they often experience residual stresses due to the fabrication process and/or working conditions. One typical example is the in-plane compressive stress in TBCs caused by the mismatch of thermal expansion coefficient between the coating and alloy substrate. Residual stresses are a major cause of film cracks and debonding. Buckling-driven delamination is a typical example of film failure under in-plane compressive residual stress, which has been extensively studied in the last few decades. Among many others, Refs [2-6] report studies on buckling-driven delamination with straight edges [2,4,6], circular edges [2,3], elliptical edges [2], and 'telephone cord'-shaped edges [5].

In studies on buckling-driven delamination, it is conventional to assume either a preexisting interface crack which is larger than the critical buckling characteristic dimension or a pre-existing imperfection [3,7,8]; however, some examples of thin-film delamination show no evidence of any pre-existing interface crack or imperfection, but still display buckling behavior [9,10]. A new hypothesis was proposed by Wang et al. [11] to explain this behavior. According to this hypothesis, delamination can be driven by pockets of energy concentration (PECs) in the form of pockets of tensile stress and shear stress, with the former being dominant $[9,10]$ on and around the interface between a thin film and a thick substrate, where PECs can be caused by a number of different processes, including thermal cooling. Based on this hypothesis, Wang et al. [11] developed an analytical mechanical model to predict several aspects of thin-film spallation failure including nucleation, stable and unstable growth, size of spallation and final kinking off. The predictions agree very well with experimental results in Refs. [9,10].

The present work aims to extend Wang et al.'s work [11] on delamination driven by PECs in two ways: First, to consider straight-edged delamination in addition to circular-edged delamination in Ref. [11]. Second, to develop analytical mechanical models for PEC-driven delamination based on the classical and the first-order shear-deformable plate mixed-modefracture partition theories [12-14] in addition to the analytical mechanical model [11] which was based only on the 2D elasticity mixed-mode-fracture partition theory [15-20]. The mechanical model for delamination with straight edges is developed in Section 2 while the model for delamination with circular edges is developed in Section 3. Theoretical predictions are compared with experimental results $[9,10]$ in Section 4. Conclusions are drawn in Section 5. 


\section{Analytical mechanical model for delamination with straight edges}

In this section, a mechanical model for delamination with straight edges is developed analytically based on the PECs hypothesis to explain several aspects of thin-film spallation failure including nucleation, stable and unstable growth, size of spallation and final kinking off. Fig. 1 shows a rectangular thin film-substrate composite material system with a throughwidth interface delamination of width $b$ and of length $2 R_{B}$. The delamination tips or the edges of the bubble are denoted by the label ' $\mathrm{B}$ '. The thickness of the film $h$ is assumed so small that only in-plane residual stresses are induced in it before delamination; and the thickness of the substrate is assumed so large that it has negligible global deformation, such as bending, extension or twisting, due to residual stresses in the film. Both the film and substrate materials are assumed to be homogeneous and isotropic. The film material has Young's modulus $E$ and Poisson's ratio $v$.

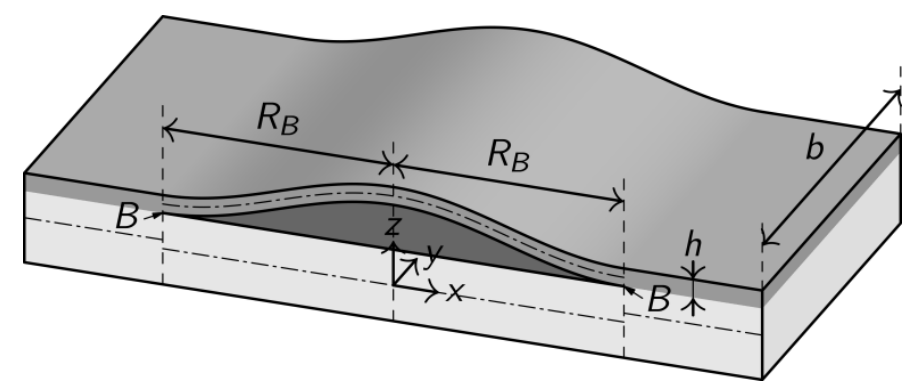

Fig. 1. A delamination bubble with straight edges.

In general, a uniaxial uniform residual stress (i.e. $\sigma_{x}=\sigma_{0}, \sigma_{y}=0, \tau_{x y}=0$ ) is achieved for a long film strip with the width of the film less than twice the thickness, and a plane stress model is suitable. Conversely, a biaxial uniform residual stress (i.e. $\sigma_{x}=\sigma_{0}, \sigma_{y}=\sigma_{0}, \tau_{x y}=0$ ) is achieved when both the width and length of the film are larger than twenty times the thickness [21,22], and a plane strain model is suitable.

\subsection{Nucleation of a delamination bubble, bubble energy and total energy release rate}

According to the PECs hypothesis, the nucleation of PEC-driven delamination is caused by pockets of tensile stress and shear stress, with the former being dominant $[9,10]$, on and around the interface. The details are unclear and are not considered in the present work. Once a delamination has nucleated, the strain energy of the stresses is freed and becomes the bottom surface energy of the delamination, the surface energy of the substrate underneath the 
bubble, and part of the strain energy in the delaminated bubble. Note that the term 'delamination bubble' is used here to differentiate it from 'delamination buckle' as the length of the bubble $2 R_{B}$ at this stage is far shorter than the critical buckling length. In order to calculate the strain energy in the bubble, its shape is approximated to be sinusoidal and represented by

$$
w(x)=\frac{A}{2}\left[1+\cos \left(\frac{\pi x}{R_{B}}\right)\right]
$$

with $w$ representing the upward deflection and $A$ the amplitude, as shown in Fig. 2. Clamped edge conditions at $x= \pm R_{B}$ are assumed because the thickness ratio between the film and the substrate is assumed very small in the present work. It is also because the local deformation of the substrate near the interface is insignificant if the Young's modulus of substrate material is greater than a third of the film's Young's modulus [21], which applies to the present case. The elastic bending strain energy can then be readily calculated using classical beam theory as

$$
U_{b}=\frac{1}{2} b \int_{-R_{B}}^{R_{B}} \frac{\bar{E} h^{3}}{12}\left(\frac{d^{2} w}{d x^{2}}\right)^{2} d x=\frac{\pi^{2}}{6} \bar{E} R_{B} h b\left(\frac{h}{R_{B}}\right)^{2}\left(\frac{\pi}{4} \frac{A}{R_{B}}\right)^{2}
$$

where $\bar{E}=E$ for plane stress conditions and $\bar{E}=E /\left(1-v^{2}\right)$ for plane strain conditions. The elastic in-plane strain energy is calculated using Hooke's law as

$$
U_{i}=2 R_{B} b h\left[u_{0}-\sigma_{0} \varepsilon_{x}^{R}+\frac{\bar{E}}{2}\left(\varepsilon_{x}^{R}\right)^{2}\right]
$$

where $u_{0}$ in Eq. (3) is the residual strain energy density in the film given by

$$
u_{0}= \begin{cases}\frac{1}{2 E} \sigma_{0}^{2} & \text { for uniaxial residual stress } \\ \frac{1-v}{E} \sigma_{0}^{2} & \text { for biaxial residual stress }\end{cases}
$$

As mentioned earlier, plane stress and plane strain models are suitable for uniaxial and biaxial stress cases respectively. The averaged axial relaxation strain in the bubble is calculated by using the conventional von Kármán geometric nonlinearity assumption.

$$
\varepsilon_{x}^{R}=\frac{1}{2 R_{B}} \int_{0}^{R_{B}}\left(\frac{d w}{d x}\right)^{2} d x=\left(\frac{\pi}{4} \frac{A}{R_{B}}\right)^{2}
$$


The averaged axial relaxation stress is then given by

$$
\sigma_{x}^{R}=\bar{E} \varepsilon_{x}^{R}=\bar{E}\left(\frac{\pi}{4} \frac{A}{R_{B}}\right)^{2}
$$

For brittle materials, the surface energy is

$$
U_{s}=b \int_{-R_{B}}^{R_{B}} G_{c}(x) d x=2 R_{B} b \bar{G}_{c}
$$

where $G_{c}(x)$ is the interface fracture toughness which is position-dependent as crack tip loading conditions vary with propagation. The fracture toughness averaged over the delaminated surface is denoted by $\bar{G}_{c}$. Note that when $A / h<<1, G_{c}(x)$ is constant, as shown later. This is the case considered in the present study. Collecting together $U_{b}, U_{i}$ and $U_{s}$ gives

$$
U=U_{0}+U_{a}
$$

where $U_{0}=2 R_{B} b h u_{0}$ is the elastic strain energy only before any separation, and $U_{a}$ is

$$
U_{a}=2 R_{B} b h \sigma_{0}\left\{\frac{1}{2 \bar{\varepsilon}_{0}}\left(\varepsilon_{x}^{R}\right)^{2}+\left[\frac{\pi^{2}}{12 \bar{\varepsilon}_{0}}\left(\frac{h}{R_{B}}\right)^{2}-1\right] \varepsilon_{x}^{R}+\frac{1}{\bar{\varphi}_{0}}\right\}
$$

which is the increase in the combined elastic strain energy and surface energy due to separation, with $\bar{\varepsilon}_{0}=\sigma_{0} / \bar{E}$ and $\bar{\varphi}_{0}=h \sigma_{0} / \bar{G}_{c}$. It can be shown that $U_{a}$ is always positive and monotonically increases with respect to the relaxation strain $\varepsilon_{x}^{R}$ or the bubble amplitude $A$ when $\left(R_{B} / h\right)^{2}<\pi^{2} /\left(12 \bar{\varepsilon}_{0}\right)$. It is seen, therefore, that a separation bubble represents another type of PEC. Its energy comes from the PEC energy 'consumed' by the bubble as it nucleates and grows. Although there is this close relationship between $U_{a}$ and the PEC energy, there are subtle differences between them. Here, $U_{a}$ is called 'bubble energy'. When the PEC energy is able to provide the bubble energy $U_{a}$ for nucleation, nucleation of a separation bubble will occur. It is expected that the bubble energy $U_{a}$ governs the growth behavior of a bubble. According to this understanding, the described bubble separation behavior is an effect of positive bubble energy; therefore, this work only considers $U_{a} \geq 0$. Obviously, when the bubble energy disappears, that is, $U_{a}=0$, a bubble will stop growing. 
Details about $U_{a}$ are given during the following development, for bubble nucleation, stable growth, unstable growth, spallation and kinking off.

After nucleation the delamination bubble bends away from the substrate, growing in height and producing a driving force for axial growth, that is, it produces energy release rate (ERR) at the bubble edge. When the ERR exceeds the interface fracture toughness, the bubble length grows. From Refs. [15,23] the total ERR $G$ is

$$
G=\frac{6}{\bar{E} h^{3}}\left(M_{x B}^{2}+\frac{h^{2}}{12} N_{x B e}^{2}\right)
$$

where $M_{x B}$ and $N_{x B e}$ are the crack tip longitudinal bending moment and effective axial force per unit width respectively, as shown in Fig. 2. They can be readily calculated in terms of $\varepsilon_{x}^{R}$ by using classical beam theory and Eq. (5), as follows:

$$
\begin{gathered}
M_{x B}=\frac{\bar{E} h^{3}}{12}\left(\frac{d^{2} w}{d x^{2}}\right)_{x=R_{B}}=\frac{2 \bar{E} h^{3}}{3 A}\left(\frac{\pi}{4} \frac{A}{R_{B}}\right)^{2}=\frac{2 \bar{E} h^{3}}{3 A} \varepsilon_{x}^{R} \\
N_{x B e}=\left[\sigma_{0}-\left(\sigma_{0}-\sigma_{x}^{R}\right)\right] h=\bar{E} h\left(\frac{\pi}{4} \frac{A}{R_{B}}\right)^{2}=\bar{E} h \varepsilon_{x}^{R}
\end{gathered}
$$

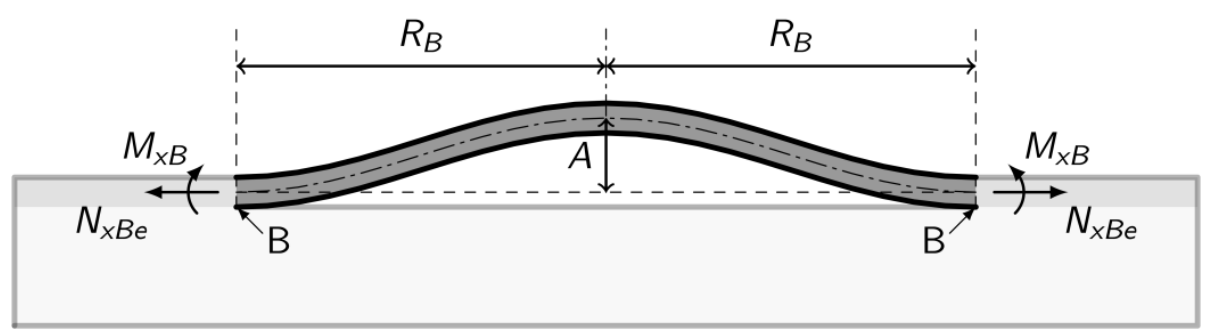

Fig. 2. Free-body diagram of a delamination bubble’s oxide film.

Substituting $M_{x B}$ from Eq. (11) and $N_{x B e}$ from Eq. (12) into Eq. (10) gives

$$
G=\frac{6 M_{x B}^{2}}{\bar{E} h^{3}}\left(1+\frac{3}{16}\left(\frac{A}{h}\right)^{2}\right)
$$

It is well known that interface fracture toughness is fracture mode mixity-dependent. Predictions of fracture toughness therefore vary with different partition theories. Refs. [2325] show, using data from extensive fracture testing [26-31], that the partition theory based on Euler beam or classical plate partition theory [12-14] gives very accurate predictions of interface fracture toughness for macroscopic mixed-mode fracture while the partition theories 
based on Timoshenko beam theory or the first-order shear-deformable plate theory [12-14] and 2D elasticity [15-20] give poor predictions. The very latest studies [11,32], however, show that the 2D elasticity partition theory gives accurate predictions for the delamination behavior of micro-scale and nano-scale thin films. This work therefore aims to develop three analytical models to predict the PEC-driven spallation behavior of thin films and to examine their respective performances. The three mechanical models are established based on these three partition theories: Euler beam or classical plate partition theory, Timoshenko beam or first-order shear-deformable plate partition theory, and 2D elasticity partition theory. After the total ERR $G$ in Eq. (13) has been partitioned into the mode I and mode II ERRs, $G_{I}$ and $G_{I I}$, a failure criterion is used to check if the delamination grows or not. In general a growth criterion can be expressed in the following form [33]:

$$
f\left(G_{I}, G_{I I}, G_{I c}, G_{I I C}\right)=0
$$

where $G_{I c}$ and $G_{\text {IIc }}$ are the respective mode I and II critical ERRs or fracture toughnesses. The form of Eq. (14) is not unique but is crack interface-dependent and is determined from experimental testing for a given interface. Many previous studies, such as those in Refs. [2325,32], have shown that the following linear propagation criterion [33] agrees with experimental results very well for brittle interfaces:

$$
\frac{G_{I}}{G_{I c}}+\frac{G_{I I}}{G_{I I c}}=\frac{1}{G_{I c}}\left(G_{I}+\frac{G_{I I}}{\psi}\right)=1 \quad \text { or } \quad G=\frac{\psi G_{I c}}{\left(G_{I} / G\right)(\psi-1)+1}=G_{c}
$$

where $\psi=G_{I I c} / G_{I c}$.

\subsection{Mechanical model based on Euler beam or classical plate partition theory}

\subsubsection{Stable growth of the delamination bubble driven by bubble energy}

Based on Euler beam or classical plate partition theory [12-14] the mode I and II ERRs for brittle interfacial fracture are

$$
G_{I E}=\frac{6 M_{x B}^{2}}{\bar{E} h^{3}}\left(1-\frac{3}{4} \frac{A}{h}\right) \quad \text { and } \quad G_{I I E}=G-G_{I E}
$$

where the subscript E denotes Euler beam or classical plate partition theory. By substituting the ERRs from Eqs. (13) and (16) into Eq. (15) with the use of Eqs. (5) and (11), the propagation criterion becomes 


$$
\left(\frac{A}{h}\right)^{2}\left[1-\left(1-\frac{1}{\psi}\right) \frac{3}{4} \frac{A}{h}+\frac{3}{16 \psi}\left(\frac{A}{h}\right)^{2}\right]=\frac{96 \bar{\varepsilon}_{0}}{\pi^{4} \varphi_{0 E}}\left(\frac{R_{B}}{h}\right)^{4}
$$

where $\varphi_{0 E}=h \sigma_{0} / G_{c E}$. Note that, consistent with the notation described above, $G_{c E}$ is the film-substrate mode-dependent interface fracture toughness $G_{c}$ based on Euler beam partition theory. It is seen from Eqs. (13) and (16) that when $A / h<<1$ then $G_{I} / G \approx 1$, that is, the delamination is pure mode I. Therefore, $G_{c E}=G_{I c}$ and $\varphi_{0 E}=h \sigma_{0} / G_{I c}$. The amplitude for crack growth is therefore obtained from Eq. (17) as

$$
\left(\frac{A}{h}\right)_{G R E}^{2}=\frac{96 \bar{\varepsilon}_{0}}{\pi^{4} \varphi_{0 E}}\left(\frac{R_{B}}{h}\right)^{4}
$$

where the subscript GR denotes growth. (Here and throughout this paper, subscripts are combined so that, for instance, GRE denotes growth based on Euler beam or classical plate partition theory.) Substituting Eq. (18) into Eqs. (4) and (5) gives the relaxation strain and stress as

$$
\begin{aligned}
& \left(\varepsilon_{x}^{R}\right)_{G R E}=\frac{6 \bar{\varepsilon}_{0}}{\pi^{2} \varphi_{0 E}}\left(\frac{R_{B}}{h}\right)^{2} \\
& \left(\sigma_{x}^{R}\right)_{G R E}=\frac{6 G_{C E}}{\pi^{2} h}\left(\frac{R_{B}}{h}\right)^{2}
\end{aligned}
$$

Note that these three quantities, $(A / h)_{G R E},\left(\varepsilon_{x}^{R}\right)_{G R E}$ and $\left(\sigma_{x}^{R}\right)_{G R E}$, are independent of the residual stress $\sigma_{0}$. The bubble energy $U_{a}$ at growth can be obtained by substituting Eq. (19) into Eq. (9).

$$
\left(U_{a}\right)_{G R E}=2 R_{B} b G_{c E}\left\{\frac{3}{2}+\frac{6 \bar{\varepsilon}_{0}}{\pi^{2}}\left(\frac{R_{B}}{h}\right)^{2}\left[\frac{3}{\pi^{2} \varphi_{0 E}}\left(\frac{R_{B}}{h}\right)^{2}-1\right]\right\}
$$

The first term in Eq. (21) is the sum of the bending strain energy and surface energy while the rest is the relaxed in-plane strain energy, which is negligible if $R_{B}$ is small due to the high powers of $R_{B}$ in these terms. The first term is therefore regarded as the nucleation energy or PEC energy required for nucleation, that is, $\left(U_{a}\right)_{N U E}=3 R_{B} b G_{c E}$ where $R_{B}$ is very small. It is seen that one third of the nucleation energy is used to bend the separation outwards after nucleating the interface delamination using two thirds of its energy. When the PEC energy is 
able to provide the bubble energy $\left(U_{a}\right)_{G R E}$, it will drive the nucleation and growth of a separation bubble. Two scenarios can occur: One scenario is slow and stable growth which occurs when $R_{B}$ is smaller than the critical buckling characteristic length. The other is unstable growth when $R_{B}$ reaches the critical value of the buckling characteristic length. The stable bubble becomes an unstable buckle. The initiation of unstable growth is considered next.

\subsubsection{Initiation of unstable growth by buckling}

During slow and stable growth, the in-plane compressive stress in the bubble $\sigma_{0}-\sigma_{x}^{R}$ reduces as its length $R_{B}$ increases. At a certain point the following condition for the buckling of thin circular plates [34] is met:

$$
\sigma_{0}-\sigma_{x}^{R}=\bar{E} \frac{(\alpha \pi)^{2}}{12}\left(\frac{h}{R_{B}}\right)^{2}
$$

where $\alpha$ is a correction factor because the buckling occurs at an amplitude $A$ which can be considered an initial imperfection; alternatively, $\alpha$ can be considered an effect of boundary conditions. The range of $\alpha$ is $0.5 \leq \alpha \leq 1.0$ with the two limits corresponding to simplysupported and clamped edge conditions respectively. A good approximation may be the average of the extreme values, that is, $\alpha=0.75$. This value is used in the present study. By using Eqs. (20) and (22), the initiation of unstable growth, which is assumed to coincide with the buckling condition, is found at

$$
\left(\frac{R_{B}}{h}\right)_{U G E}^{2}=\frac{\pi^{2} \varphi_{0 E}}{12}\left[1-\left(1-\frac{\alpha^{2}}{\Omega_{E}}\right)^{1 / 2}\right]
$$

with the subscript UG denoting the initiation of unstable growth and

$$
\Omega_{E}=\frac{1}{2} \bar{\varepsilon}_{0} \varphi_{0 E}=\frac{h \sigma_{0}^{2}}{2 \bar{E} G_{c E}}
$$

There is no unstable growth when $\Omega_{E}<\alpha^{2}$. Binomial expansion of the expression in the square bracket in Eq. (23) for $\Omega_{E}>\alpha^{2}$, leads to

$$
\left(\frac{R_{B}}{h}\right)_{U G E}^{2}=\frac{(\alpha \pi)^{2}}{12 \bar{\varepsilon}_{0}}
$$


Substituting Eq. (25) into Eqs. (18), (19) and (20), and use of Eq. (24) where appropriate, results in Eqs. (26), (27) and (28), respectively.

$$
\begin{gathered}
\left(\frac{A}{h}\right)_{U G E}^{2}=\frac{\alpha^{4}}{3 \Omega_{E}} \\
\left(\varepsilon_{x}^{R}\right)_{U G E}=\frac{\alpha^{2}}{2 \varphi_{E 0}} \\
\left(\sigma_{x}^{R}\right)_{U G E}=\frac{\alpha^{2} \bar{E}}{2 \varphi_{0 E}}=\frac{\alpha^{2} G_{c E} \bar{E}}{2 h \sigma_{0}}=\frac{\alpha^{2} \sigma_{0}}{4 \Omega_{E}}
\end{gathered}
$$

The bubble energy at the initiation of unstable growth when $\Omega_{E}>\alpha^{2}$ is obtained by substituting Eq. (25) into Eq. (21) and using Eq. (24).

$$
\left(U_{a}\right)_{U G E}=2\left(R_{B}\right)_{U G E} b G_{c E}\left(\frac{3-\alpha^{2}}{2}+\frac{\alpha^{4}}{16 \Omega_{E}}\right) \approx\left(3-\alpha^{2}\right)\left(R_{B}\right)_{U G E} b G_{c E}=\frac{\left(3-\alpha^{2}\right) \alpha \pi b h G_{c E}}{2 \sqrt{3 \bar{\varepsilon}_{0}}}
$$

\subsubsection{Unstable growth and spallation of the buckle driven by buckling and bubble energy}

The developments in the section are generally approximate due to neglect of the dynamic effect of abrupt unstable growth and the effect of large amplitude $A$. Some more detailed discussions on this will be given later in this section and in Section 4. Since the bubble energy $U_{a}$ governs the growth behavior of the separation, the variation of bubble energy at growth $\left(U_{a}\right)_{G R}$ in Eq. (25) is considered. By differentiating $\left(U_{a}\right)_{G R}$ in Eq. (21) with respect to $R_{B} / h$, its maximum is found to occur at

$$
\left(\frac{R_{B}}{h}\right)_{M U E}^{2}=\frac{\pi^{2} \varphi_{0 E}}{10}\left[1-\left(1-\frac{5}{6 \Omega_{E}}\right)^{1 / 2}\right]
$$

with the subscript MU denoting the maximum $\left(U_{a}\right)_{G R E}$. When $\Omega_{E}<5 / 6$ there is no solution. Binomial expansion of the expression in the square bracket in Eq. (30) for $\Omega_{E}>>5 / 6$, leads to

$$
\left(\frac{R_{B}}{h}\right)_{M U E}^{2}=\frac{\pi^{2}}{12 \bar{\varepsilon}_{0}}
$$

Substituting Eq. (31) into Eqs. (18), (19) and (20), and use of Eq. (24) where appropriate, results in Eqs. (32), (33) and (34), respectively. 


$$
\begin{gathered}
\left(\frac{A}{h}\right)_{M U E}^{2}=\frac{1}{3 \Omega_{E}} \\
\left(\varepsilon_{x}^{R}\right)_{M U E}=\frac{1}{2 \varphi_{0 E}} \\
\left(\sigma_{x}^{R}\right)_{M U E}=\frac{\bar{E}}{2 \varphi_{0 E}}=\frac{G_{C E} \bar{E}}{2 h \sigma_{0}}=\frac{\sigma_{0}}{4 \Omega_{E}}
\end{gathered}
$$

Note that $\left(R_{B}\right)_{M U E}$ in Eq. (31) is equal to $\left(R_{B}\right)_{U G E}$ in Eq. (25) with $\alpha=1.0$ for a clamped edge condition. Substituting Eq. (31) into Eq. (21) gives the bubble energy when $\Omega_{E}>>5 / 6$ as

$$
\left(U_{a}\right)_{M U E}=2\left(R_{B}\right)_{M U E} b G_{c E}\left(1+\frac{1}{16 \Omega_{E}}\right) \approx 2\left(R_{B}\right)_{M U E} b G_{c E}=\frac{\pi b h G_{c E}}{\sqrt{3 \bar{\varepsilon}_{0}}}
$$

More importantly, $\left(U_{a}\right)_{G R E}$ becomes zero at

$$
\left(\frac{R_{B}}{h}\right)_{S P E}^{2}=\frac{\pi^{2} \varphi_{0 E}}{6}\left\{1-\left[1-\frac{3}{2 \Omega_{E}}\right]^{1 / 2}\right\}
$$

When $\Omega_{E}<3 / 2$ there is no solution. Binomial expansion of the expression in the square bracket in Eq. (40) for $\Omega_{E}>>3 / 2$, leads to

$$
\left(\frac{R_{B}}{h}\right)_{S P E}^{2}=\frac{\pi^{2}}{4 \bar{\varepsilon}_{0}}
$$

Substituting Eq. (37) into Eqs. (18), (19) and (20), and use of Eq. (24) where appropriate, results in Eqs. (38), (39) and (40), respectively.

$$
\begin{gathered}
\left(\frac{A}{h}\right)_{S P E}^{2}=\frac{3}{\Omega_{E}} \\
\left(\varepsilon_{x}^{R}\right)_{S P E}=\frac{3}{2 \varphi_{0 E}} \\
\left(\sigma_{x}^{R}\right)_{S P E}=\frac{3 \bar{E}}{2 \varphi_{0 E}}=\frac{3 G_{c E} \bar{E}}{2 h \sigma_{0}}=\frac{3 \sigma_{0}}{4 \Omega_{E}}
\end{gathered}
$$

At this moment the unstable growth stops as there is no driving energy; however, if the kinetic energy due to fast unstable growth of the buckle is large enough to break the film at its edge, the buckle spalls. The subscript SP in the equations above denotes spallation. 
A sketch of the variation of $\left(U_{a}\right)_{G R E}$ in Eq. (21) is now given in Fig. 3, based on the preceding analysis. Note that the sketch of the variation of $\left(U_{a}\right)_{G R E}$ for a delamination with a circular edge is based on Eq. (53) and the analysis in Section 3. The bubble energy $\left(U_{a}\right)_{G R E}$, given by Eq. (21), increases with growth up to $\left(R_{B}\right)_{M U E}$, given by Eq. (30) and approximately by Eq. (31). This increase comes from the PEC. Because of the increasing nature of the bubble energy $\left(U_{a}\right)_{G R E}$ in this region, the growth is expected to be generally slow and steady, even in the first range of unstable growth, that is, in the range $\left(R_{B}\right)_{U G E} \leq R_{B} \leq\left(R_{B}\right)_{M U E}$. Unstable growth starts at $\left(R_{B}\right)_{U G E}$, given by Eq. (23) and approximately by Eq. (25). In the first region of unstable growth, the bubble length grows by a factor of about $1 / \alpha=1.333$, and the amplitude by a factor of about $1 / \alpha^{2}=1.778$. When the PEC is too weak to provide the bubble energy, the bubble will stop growing, even in the first unstable growth range.

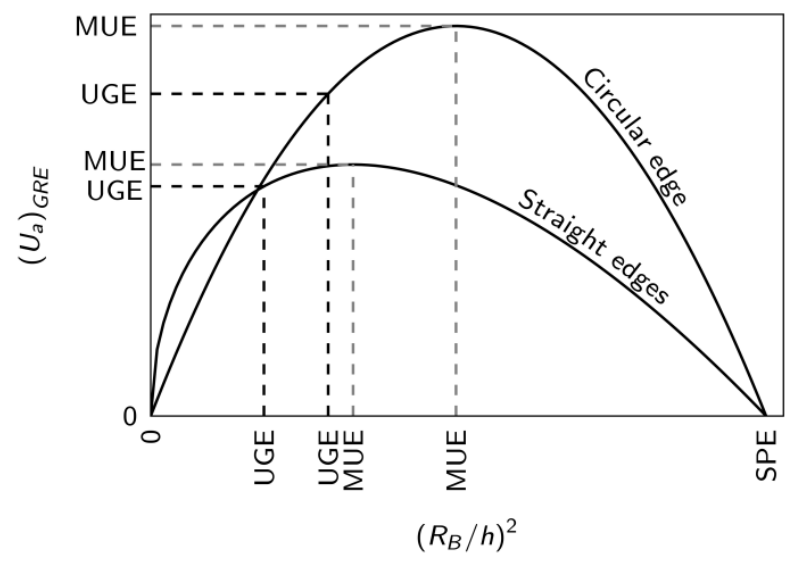

Fig. 3. Sketch of the variation of $\left(U_{a}\right)_{G R E}$ with respect to $\left(R_{B} / h\right)^{2}$ using Eq. (21) for a delamination with straight edges and Eq. (53) for a delamination with a circular edge.

The bubble energy $\left(U_{a}\right)_{G R E}$ decreases with growth after $\left(R_{B}\right)_{M U E}$ and reduces to zero at $\left(R_{B}\right)_{S P E}$, given by Eq. (36) and approximately by Eq. (37). This decreasing nature has two meanings. The first is that the bubble is no longer able to store any further bubble energy from the PEC. The second is that the bubble energy $\left(U_{a}\right)_{M U E}$, cumulated in the range $0 \leq R_{B} \leq\left(R_{B}\right)_{M U E}$, is being transformed into kinetic energy. This is consistent with the fact that the bifurcation-type buckling occurs at around $\left(R_{B}\right)_{M U E}$, resulting in more 'violent' growth after $\left(R_{B}\right)_{M U E}$. In this second region of unstable growth, that is, in the range $\left(R_{B}\right)_{M U E} \leq R_{B} \leq\left(R_{B}\right)_{S P E}$, the bubble length grows by a factor of about 1.732 , and the 
amplitude by a factor of about 3. Obviously, the bubble will stop growth at $\left(R_{B}\right)_{S P E}$ as the bubble energy becomes zero. The minimum kinetic energy can be estimated as $\left(U_{a}\right)_{M U E}$ in Eq. (35) by assuming that the PEC boundary ends at $\left(R_{B}\right)_{M U E}$ resulting in no further contribution to the kinetic energy. When $\left(U_{a}\right)_{M U E}$ is large enough to break the film, then spallation occurs, that is, the interface crack kinks into the film. The kink-off angle $\beta$ is measured from the interface as shown in Fig. 4.

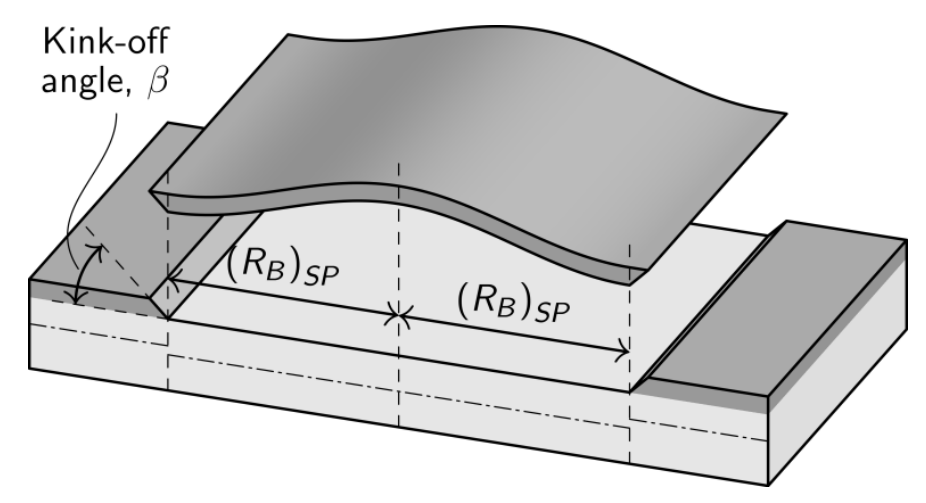

Fig. 4. The kink-off angle of a straight-edged film spall.

The kink-off angle can be determined using

$$
2\left(R_{B}\right)_{M U E} b G_{c E}=\frac{2 b h}{\sin (\beta)} G_{c f}
$$

where $G_{c f}$ is the fracture toughness of the film material, which is generally different to the fracture toughness of the film-substrate interface $G_{c E}$. Note that the left-hand side of Eq. (41) comes from Eq. (35) and the right-hand side is the breaking surface energy of the oxide film as shown in Fig. 4. The kink-off angle is then obtained from Eq. (41) as follows, after substituting $\left(R_{B}\right)_{M U E}$ and $\left(R_{B}\right)_{S P E}$ from Eqs. (31) and (37) respectively:

$$
\beta_{E}=\arcsin \left(\frac{2 \sqrt{3 \bar{\varepsilon}_{0}}}{\pi} \frac{G_{c f}}{G_{c E}}\right)
$$

\subsection{Mechanical model based on Timoshenko beam or first-order shear-deformable plate partition theory}

Since the development in this section closely follows that in Section 2.2, only several key equations are recorded here. Based on Timoshenko beam theory or first-order sheardeformable plate theory [12-14] the mode I and II ERRs are 


$$
G_{I T}=\frac{6 M_{x B}^{2}}{4 \bar{E} h^{3}}\left(1-\frac{3}{4} \frac{A}{h}\right)^{2} \quad \text { and } \quad G_{I I T}=G-G_{I T}
$$

where the subscript $\mathrm{T}$ denotes Timoshenko beam or first-order shear-deformable plate partition theory. It is seen from Eqs. (13) and (43) that when $A / h<<1$ then $G_{I} / G \approx 0.25$, that is, the delamination is mode-II-dominant. The total critical ERR $G_{c T}$ is then found by using Eqs. (13), (15) and (43) as

$$
G_{c T}=\frac{4 \psi}{3+\psi} G_{I c}=\lambda_{T} G_{I c}=\lambda_{T} G_{c E}
$$

where

$$
\lambda_{T}=\frac{4 \psi}{3+\psi}
$$

This gives

$$
\varphi_{0 T}=\frac{h \sigma_{0}}{G_{c T}}=\frac{\varphi_{0 E}}{\lambda_{T}} \quad \text { and } \quad \Omega_{T}=\frac{1}{2} \bar{\varepsilon}_{0} \varphi_{0 T}=\frac{\Omega_{E}}{\lambda_{T}}
$$

The mechanical model can be readily obtained by replacing $G_{c E}, \varphi_{0 E}$ and $\Omega_{E}$ in the mechanical model based on the Euler beam or classical plate partition theories with $G_{c T}, \varphi_{0 T}$ and $\Omega_{T}$ respectively. Since $G_{c T}$ is usually larger than $G_{c E}$ (since usually $G_{\text {IIc }}>G_{I C}$ ) then $\varphi_{0 T}$ and $\Omega_{T}$ are usually smaller than $\varphi_{0 E}$ and $\Omega_{E}$ respectively. These differences result in larger values for $A / h, \varepsilon_{x}^{R}$ and $\sigma_{x}^{R}$; however, it is interesting to note that when $\Omega_{T}$ is large enough, the values of $R_{B} / h$ at the initiation of unstable growth, the maximum of PEC energy and final spallation are the same as those from the mechanical model based on Euler beam or classical plate partition theory.

\subsection{Mechanical model based on 2 D elasticity partition theory}

Similarly, 2D elasticity partition theory [15-20] gives the mode I and II ERRs as

$$
G_{I 2 D}=0.6227 \frac{6 M_{x B}^{2}}{\bar{E} h^{3}}\left(1-\frac{30}{89} \frac{A}{h}\right)^{2} \quad \text { and } \quad G_{I I 2 D}=G-G_{I 2 D}
$$


where the subscript 2D denotes 2D elasticity partition theory. It is seen from Eqs. (13) and (47) that when $A / h<<1$ then $G_{I} / G \approx 0.6227$, that is, the delamination is mixed-mode. The total critical ERR $G_{c 2 D}$ is then found by using Eqs. (13), (15) and (47) as

$$
G_{c 2 D}=\frac{\psi}{0.3773+0.6227 \psi} G_{I c}=\lambda_{2 D} G_{I c}=\lambda_{2 D} G_{c E}
$$

where

$$
\lambda_{2 D}=\frac{\psi}{0.3773+0.6227 \psi}
$$

This gives

$$
\varphi_{02 D}=\frac{h \sigma_{0}}{G_{c 2 D}}=\frac{\varphi_{0 E}}{\lambda_{2 D}} \quad \text { and } \quad \Omega_{2 D}=\frac{1}{2} \bar{\varepsilon}_{0} \varphi_{02 D}=\frac{\Omega_{E}}{\lambda_{2 D}}
$$

The mechanical model can be readily obtained by replacing $G_{c E}, \varphi_{0 E}$ and $\Omega_{E}$ in the mechanical model based on the Euler beam or classical plate partition theories with $G_{c 2 D}$, $\varphi_{02 D}$ and $\Omega_{2 D}$ respectively. Similar to in Section 2.3, $G_{c 2 D}$ is usually larger than $G_{c E}$ (since usually $G_{I I c}>G_{I c}$ ) and therefore $\varphi_{02 D}$ and $\Omega_{2 D}$ are usually smaller than $\varphi_{0 E}$ and $\Omega_{E}$ respectively, resulting in larger values for $A / h, \varepsilon_{x}^{R}$ and $\sigma_{x}^{R}$. If $\Omega_{2 D}$ is large enough, however, the values of $R_{B} / h$ at the initiation of unstable growth, the maximum of PEC energy and final spallation are the same as those from the mechanical model based on Euler beam or classical plate partition theory.

\section{Analytical mechanical model for delamination with a circular edge}

The mechanical development in this section closely follows that in Section 2 for delamination with straight edges. Only the key developments are therefore recorded here. Fig. 5 shows circular separation bubble of radius $R_{B}$. The delamination tips or the edge of the bubble are denoted by the label ' $\mathrm{B}$ '. The model is developed in a polar coordinate system. Biaxial compressive residual stress is assumed (i.e. $\sigma_{r}=\sigma_{\theta}=\sigma_{0}$ ) so the model is effectively plane strain with $\bar{E}=E /\left(1-v^{2}\right)$. 


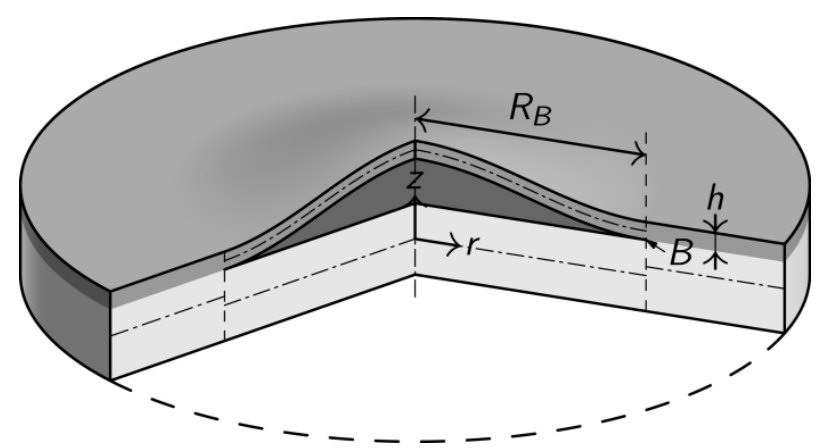

Fig. 5. A delamination bubble with a circular edge.

\subsection{Nucleation of a delamination bubble, bubble energy and total energy release rate}

The bubble shape is approximated to be axisymmetric and in the form,

$$
w(r)=\frac{A}{2}\left[1+\cos \left(\frac{\pi r}{R_{B}}\right)\right]
$$

The bubble energy is calculated as

$$
U_{a}=\pi R_{B}^{2} h \sigma_{0}\left\{\frac{1}{2 \bar{\varepsilon}_{0}}\left(\varepsilon_{r}^{R}\right)^{2}+\left[\frac{\pi^{2}}{12 \bar{\varepsilon}_{0}}\left(\frac{h}{R_{B}}\right)^{2}-1\right] \varepsilon_{r}^{R}+\frac{1}{\bar{\varphi}_{0}}\right\}
$$

Note the similarity between the bubble energy in Eq. (9) for a straight-edged bubble and in Eq. (52) for a circular-edged bubble. The equations for total ERR are identical to those in Eqs. (10) to (13), except with the $x$-coordinate swapped for the $r$-coordinate. The linear failure criterion in Eq. (15) is used again. The following development is based on classical plate partition theory [12-14]. The mechanical models based on the first-order sheardeformable plate and the 2D elasticity partition theories can be readily obtained by using the same parameter replacements as for the straight-edged case, as described in Sections 2.3 and 2.4 respectively.

\subsection{Stable growth of the delamination bubble driven by bubble energy}

There are no changes to $(A / h)_{G R E},\left(\varepsilon_{r}^{R}\right)_{G R E}$ and $\left(\sigma_{r}^{R}\right)_{G R E}$ in Eqs. (16) to (20) except for swapping the subscript $x$-coordinate for the $r$-coordinate. The bubble energy at growth in Eq. (21) changes to be

$$
\left(U_{a}\right)_{G R E}=\pi R_{B}^{2} G_{c E}\left\{\frac{3}{2}+\frac{6 \bar{\varepsilon}_{0}}{\pi^{2}}\left(\frac{R_{B}}{h}\right)^{2}\left[\frac{3}{\pi^{2} \varphi_{0 E}}\left(\frac{R_{B}}{h}\right)^{2}-1\right]\right\}
$$


A sketch of the variation of $\left(U_{a}\right)_{G R E}$ for a delamination with a circular edge is also given in Fig. 3.

\subsection{Initiation of unstable growth by buckling}

There are no changes to $\left(R_{B} / h\right)_{U G E},(A / h)_{U G E},\left(\varepsilon_{r}^{R}\right)_{U G E}$ and $\left(\sigma_{r}^{R}\right)_{U G E}$ in Eqs. (23) and (25) to (28) except for swapping the subscript $x$-coordinate for the $r$-coordinate. Also, now the range of $\alpha$ is $0.652 \leq \alpha \leq 1.22$ with the two limits corresponding to simply-supported and clamped edge conditions respectively. The average value $\alpha=0.936$ is used in the present study. The bubble energy at the initiation of unstable growth when $\Omega_{E}>\alpha^{2}$ is

$$
\left(U_{a}\right)_{U G E}=\pi\left(R_{B}\right)_{U G E}^{2} G_{c E}\left(\frac{3-\alpha^{2}}{2}+\frac{\alpha^{4}}{16 \Omega_{E}}\right) \approx \frac{3-\alpha^{2}}{2} \pi\left(R_{B}\right)_{U G E}^{2} G_{c E}=\frac{\left(3-\alpha^{2}\right) \alpha^{2} \pi^{3} h^{2} G_{c E}}{24 \bar{\varepsilon}_{0}}
$$

3.4. Unstable growth and spallation of the buckle driven by buckling and bubble energy

The maximum bubble energy occurs at

$$
\left(\frac{R_{B}}{h}\right)_{M U E}^{2}=\frac{\pi^{2} \varphi_{0 E}}{9}\left[1-\left(1-\frac{9}{8 \Omega_{E}}\right)^{1 / 2}\right]
$$

When $\Omega_{E}<9 / 8$ there is no solution. Binomial expansion of the expression in the square bracket in Eq. (55) for $\Omega_{E}>>9 / 8$, leads to

$$
\left(\frac{R_{B}}{h}\right)_{M U E}^{2}=\frac{\pi^{2}}{8 \bar{\varepsilon}_{0}}
$$

Substituting Eq. (56) into Eqs. (18), (19) and (20) with the subscript $x$-coordinate swapped for the $r$-coordinate, and use of Eq. (24) where appropriate, results in Eqs. (57), (58) and (59), respectively.

$$
\begin{gathered}
\left(\frac{A}{h}\right)_{M U E}^{2}=\frac{3}{4 \Omega_{E}} \\
\left(\varepsilon_{r}^{R}\right)_{M U E}=\frac{3}{4 \varphi_{E 0}} \\
\left(\sigma_{r}^{R}\right)_{M U E}=\frac{3 \bar{E}}{4 \varphi_{0 E}}=\frac{G_{c E} \bar{E}}{4 h \sigma_{0}}=\frac{3 \sigma_{0}}{8 \Omega_{E}}
\end{gathered}
$$


Note that $\left(R_{B}\right)_{M U E}$ in Eq. (56) is equal to $\left(R_{B}\right)_{U G E}$ in Eq. (25) with $\alpha=1.220$ for a circular buckle with a clamped edge condition. Substituting Eq. (56) into Eq. (52) gives the maximum bubble energy when $\Omega_{E}>>9 / 8$ as

$$
\left(U_{a}\right)_{M U E}=\pi\left(R_{B}\right)_{M U E}^{2} G_{c E}\left(\frac{3}{4}+\frac{9}{64 \Omega_{E}}\right) \approx \frac{3}{4} \pi\left(R_{B}\right)_{M U E}^{2} G_{c E}=\frac{3 \pi^{3} h^{2} G_{c E}}{32 \bar{\varepsilon}_{0}}
$$

Eqs. (36) to (40) for spallation remain the same except for swapping the subscript $x$ coordinate for the $r$-coordinate. The kink-off angle is measured from the interface as shown in Fig. 6.

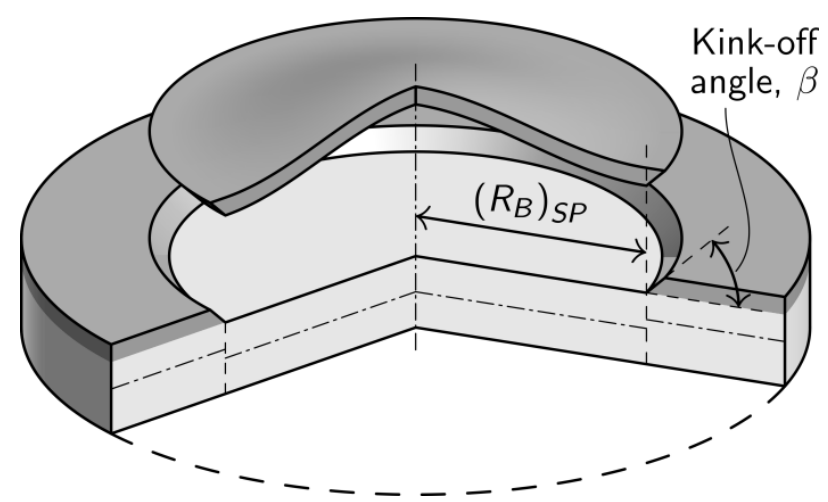

Fig. 6. The kink-off angle of a circular-edged film spall.

The kink-off angle can be determined using

$$
\frac{3}{4} \pi\left(R_{B}\right)_{M U E}^{2} G_{c E}=\frac{2 \pi R_{B S P} h}{\sin (\beta)} G_{c f}
$$

where $G_{c f}$ is the fracture toughness of the oxide film. Note that the left-hand side of Eq. (61) comes from Eq. (60) and the right-hand side is the breaking surface energy of the oxide film as shown in Fig. 6. The kink-off angle is then obtained from Eq. (61) as follows, after substituting $\left(R_{B}\right)_{M U E}$ and $\left(R_{B}\right)_{S P E}$ from Eqs. (56) and (37) respectively:

$$
\beta_{E}=\arcsin \left(\frac{32 \sqrt{\bar{\varepsilon}_{0}}}{3 \pi} \frac{G_{c f}}{G_{c E}}\right)
$$

The mechanical models based on first-order shear-deformable plate partition theory and 2D elasticity partition theory can be readily obtained by replacing $G_{c E}, \varphi_{0 E}$ and $\Omega_{E}$ in the 
mechanical model based on classical plate partition theory with $G_{c T}, \varphi_{0 T}$ and $\Omega_{T}$, and $G_{c 2 D}$, $\varphi_{02 D}$ and $\Omega_{2 D}$ respectively.

Some salient points are now summarized. When using classical plate partition theory and assuming $A / h<<1$, the whole delamination process (i.e. the nucleation, stable and unstable growth, and final spallation) for both straight-edged and circular-edged delaminations is a pure mode I fracture. Both also have the same nucleation bubble energy intensity, that is, $1.5 G_{c E}$, from Eqs. (21) and (53). Unstable growth for both starts at $\left(R_{B} / h\right)^{2}=(\alpha \pi)^{2} /\left(12 \bar{\varepsilon}_{0}\right)$ from Eq. (25) with a bubble energy intensity of $\left(3-\alpha^{2}\right) G_{c E} / 2$ from Eqs. (29) and (54). (With the buckling correction factor $\alpha=0.75$ for straight-edged delamination and $\alpha=0.936$ for

circular-edged.) The maximum bubble energy occurs at $\left(R_{B} / h\right)^{2}=\pi^{2} /\left(12 \bar{\varepsilon}_{0}\right)$ for straight edges with the bubble energy intensity $G_{c E}$ from Eq. (35); and at $\left(R_{B} / h\right)^{2}=\pi^{2} /\left(8 \bar{\varepsilon}_{0}\right)$ for a circular edge with the bubble energy intensity $0.75 G_{c E}$ from Eq. (61). Spallation occurs at $\left(R_{B} / h\right)^{2}=\pi^{2} /\left(4 \bar{\varepsilon}_{0}\right)$ for both straight- and circular-edged delaminations when the bubble energy is zero. Note that the bubble energy intensity decreases during delamination growth as expected; however, it is interesting to note that at the start of unstable growth and at the maximum bubble energy radius, the bubble energy intensities for straight-edged delamination are larger than those for a circular-edged delamination. This suggests that circular spallation occurs more easily than straight-edged spallation, as usually expected. When first-order shear-deformable plate or 2D elasticity partition theory is used, the whole delamination process is a mixed-mode fracture. In the next section, the mechanical development in this section will be assessed by comparing its predictions with experimental results $[9,10]$.

\section{Experimental comparison}

In this section, predictions from the mechanical models above are compared against the remarkable and thought-provoking experimental results from the excellent studies by Tolpygo and Clarke $[9,10]$ on the room temperature spallation of $\alpha$-alumina films grown by oxidation. Wang et al.'s work [11] and the present study were both triggered by the Tolpygo and Clarke's studies [9,10]. Ref. [11] examined the model above in Section 3 for circularedged delamination based on 2D elasticity partition theory and found excellent agreement between the model's predictions and experimental results. This work extends this comparison 
to the mechanical models based on the classical plate and the first-order shear-deformable plate partition theories.

It is believed that an introduction to the studies by Tolpygo and Clarke $[9,10]$ is helpful to understand the present work. In these studies, $\alpha-\mathrm{Al}_{2} \mathrm{O}_{3}$ films of different thicknesses were formed on the surface of Fe-Cr-Al heat-resistant alloy substrates of different thicknesses by oxidizing them at $1200^{\circ} \mathrm{C}$ for different time periods. Then, the film-substrate material systems were cooled to room temperature at different cooling rates. Interestingly, no spallation failure was observed during cooling at any rate, during which compressive residual stress gradually increases due to thermal expansion mismatch. Surprisingly, however, for some cooling rates, it was observed that circular interfacial separations between the film and the substrate nucleate, grow in separation distance and propagate radially, all after reaching room temperature, at a constant compressive residual stress far below the critical buckling stress, and apparently spontaneously. After a period of slow and stable growth, some of these separations then grow abruptly and the oxide spalls off.

Various explanations for the phenomenon were proposed and thoroughly and insightfully examined by Tolpygo and Clarke [9,10]. One category of these explanations was the flaw or imperfection hypothesis, which attempted to explain the nucleation and growth of the separations. The hypothesized flaw included pre-existing separations, cavities and other large defects, and impurity segregations at the oxide-substrate interface due to the slow cooling rates. Optical microscopy studies, however, showed that no discernible interfacial separations or spallation existed in any of the specimens when examined immediately after cooling to room temperature. Also, when examining the exposed metal surface after spallation, scanning electron microscopy studies did not reveal any interfacial cavities or voids except for areas near sharp edges at the periphery of the specimens. This flaw hypothesis was therefore invalidated. In their second hypothesis, the time-dependent growth behavior of the separations was explained by stress corrosion due to moisture. To have a convincing invalidation of this hypothesis, some slowly-cooled specimens were placed in a sealed container in a purified nitrogen atmosphere with zero humidity. Spallation was still as prevalent as during regular exposure in ambient atmosphere. In addition, several other hypotheses were also considered, such as condensation of equilibrium thermal vacancies at the interface during cooling, diffusion of hydrogen or carbon monoxide from the metal to the film causing disruption to the film at room temperature, and metal embrittlement or hardening near the interface. Tolpygo and Clarke, however, stated that none of these hypotheses was consistent with all the experimental results. Readers are strongly 
recommended to read their work $[9,10]$ for a thorough understanding of the above descriptions.

Wang et al.'s work [11] and the present study both hypothesize that PECs in the filmmetal material system may be the cause of film separation and spallation at constant in-plane compressive stress after cooling to room temperature. Pockets of tensile stress and shear stress on and around the interface are formed during cooling and are randomly distributed. Pockets of interface stresses result in PECs and these PECs cause interface separation nucleation, growth and spallation of the film.

Fig. 7 shows a sequence of optical images from Ref. [10] illustrating the nucleation, growth and spallation of one typical separation from a specimen where the oxide thickness is $8 \mu \mathrm{m}$ after 100 -h oxidation at $1200^{\circ} \mathrm{C}$ and the residual compressive stress measured far away from spalls is $4.3 \mathrm{GPa}$ due to cooling at $50^{\circ} \mathrm{C} \mathrm{min}$. Figs. 6a-e show the nucleation and stable growth stages which took $120 \mathrm{~min}$. The time values were counted from when the specimen just reached room temperature from cooling. In Figs. 6a-d the radius of the nearly circular separation is far below the critical value for buckling. In Ref. [10], this type of separation is called an 'incipient buckle'. Here it is called a 'separation bubble' because its radius is far too small to cause buckling, particularly in Figs. 6a-c. The abrupt growth starts after Fig. 7e and spallation failure has occurred by Fig. 7f, taking less than $4 \mathrm{~min}$. The following aims to test the mechanical models developed in Sections 2 and 3 to explain some aspects of the above observations. 

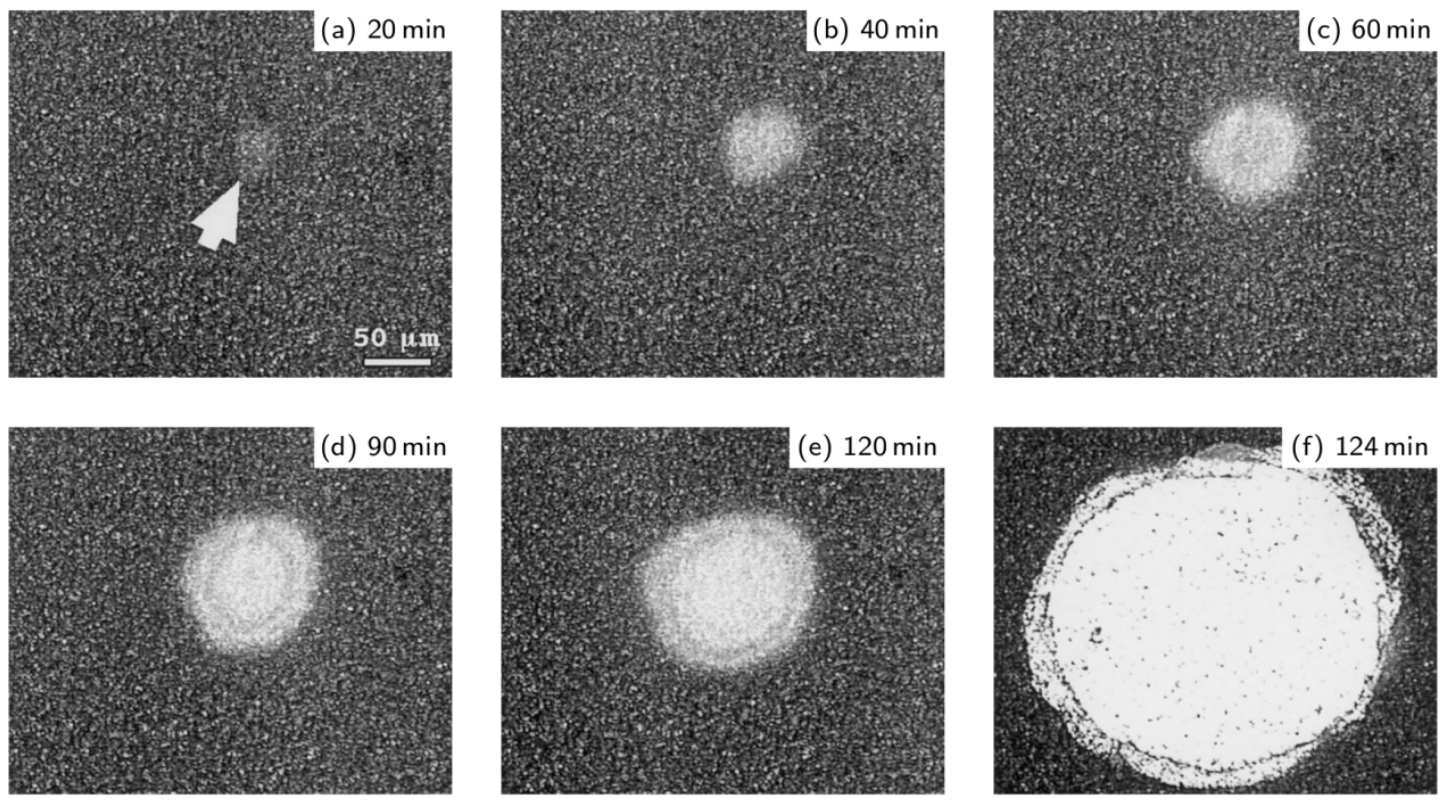

Fig. 7. A sequence of optical images showing the nucleation and growth of a separation bubble with time at room temperature leading to sudden spallation after $124 \mathrm{~min}$. Reprinted with permission from Tolpygo, V.K., Clarke, D.R., 2000. Mater. Sci. Eng. A278, 151-161. Copyright 2000 Elsevier.

In the following comparisons, the material properties of the oxide film are taken from the Tolpygo and Clarke's studies [9,10], and are as follows: The Young's modulus is $E=400 \mathrm{GPa}$ and the Poisson's ratio is $v=0.25$. The mode I critical ERR of the interface is $G_{I c}=8.6 \mathrm{~N} / \mathrm{m}$ and the critical mode I ERR of the oxide film is $G_{c f}=20 \mathrm{~N} / \mathrm{m}$. The ratio $\psi=G_{\text {IIc }} / G_{\text {Ic }}=5$ is used, which, based on Ref. [35], is considered by the authors to be a representative value.

First, Eq. (25) is used to predict the initiation of unstable growth, and Eq. (37) is used to predict the size of spallation. The solid dots in Fig. 8 represent a series of measurements of the size of individual separations as a function of time at room temperature. The time of 0 min corresponds to the moment when the specimen was placed under the microscope and its temperature was close to ambient. 
(a) $h=4.9 \mu \mathrm{m}, \sigma_{0}=4.46 \mathrm{GPa}$

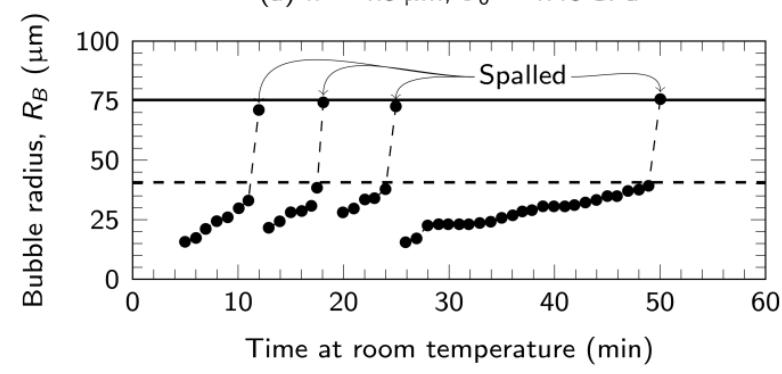

(b) $h=6.2 \mu \mathrm{m}, \sigma_{0}=4.45 \mathrm{GPa}$

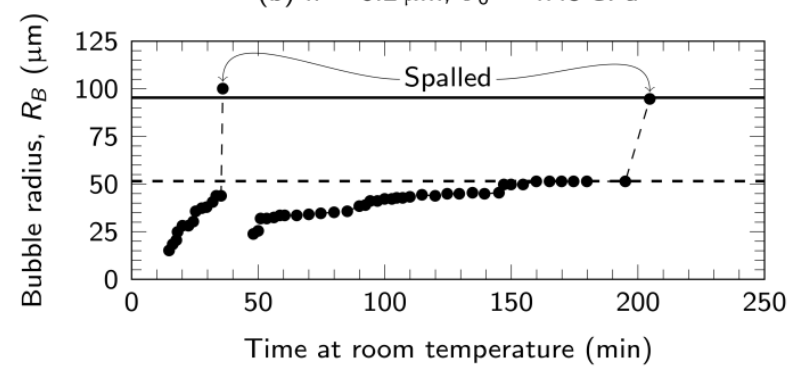

(c) $h=8.0 \mu \mathrm{m}, \sigma_{0}=4.31 \mathrm{GPa}$

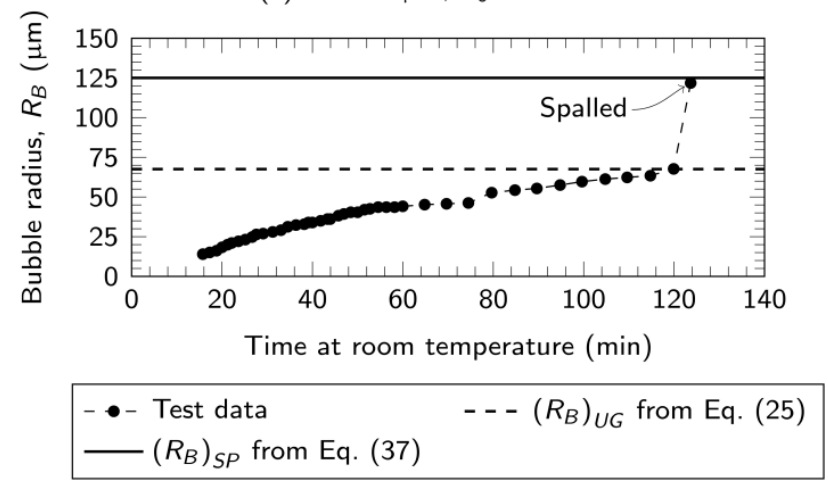

Fig. 8. Separation bubble radius versus time at room temperature for three different samples [10].

Fig. 8a shows data from four different separation bubbles on a single specimen after isothermal oxidation for $25 \mathrm{~h}$ at $1200^{\circ} \mathrm{C}$ and cooling at $20^{\circ} \mathrm{C} \mathrm{min}{ }^{-1}$. The bubbles were successively monitored using optical microscopy. All of the bubbles grew at a constant compressive stress of $\sigma_{0}=4.46 \pm 0.4 \mathrm{GPa}$, which was measured in the adherent oxide far away from the separations. The whole process includes nucleation, stable growth, unstable growth, and final spallation. The nucleation of separation bubbles was not recorded due to the difficulty of making timely observations of nucleating bubbles using this monitoring technique. Stable growth, however, with a radius far smaller than the critical buckling value, was readily observed. At a certain critical radius, which is again far smaller than the critical buckling radius, unstable growth abruptly occurs and final spallation takes place. It is pertinent that all four separations start unstable growth at approximately the same radius, and 
then all eventually spall off also at approximately the same radius. Two specimens with thicker oxide layers were produced with $50 \mathrm{~h}$ and $100 \mathrm{~h}$ of oxidation and are shown in Figs. $7 \mathrm{~b}$ and c respectively. The growth behaviors of two separation bubbles are shown in Fig. 8b. Again, the two separations start unstable growth at approximately the same radius, and then both eventually spall off also at approximately the same radius. Fig. 8c shows the growth behavior of one separation bubble. The horizontal lines in Figs. 7a-c represent the predictions from Eq. (25) for the initiation of unstable growth and Eq. (37) for final spallation. It is very impressive to see that the predictions from Eqs. (25) and (37) have excellent agreement with the test results. It is worth noting that Eqs. (25) and (37) are common to all three mechanical models (based on the classical plate, first-order shear-deformable plate and 2D elasticity partition theories) as long as the value of the parameter $\Omega$ meets the requirements of Eqs. (25) and (37).

To examine the accuracy of the approximate Eqs. (25) and (37), Table 1 compares their predictions with those from Eqs. (23) and Eq. (36) respectively. SP, 2D and CP represent the mechanical models based on first-order shear-deformable plate partition theory, the 2D elasticity partition theory and the classical plate partition theory respectively. It is seen that they agree with each other very well. The ratio $\psi=G_{\text {IIc }} / G_{I c}=5$ is used to calculate SP and 2D predictions.

Table 1. Comparison of the present mechanical model with test data [10] for the initiation of unstable growth, the size of spallation and kinking off.

\begin{tabular}{|c|c|c|c|c|c|c|c|c|c|c|c|c|c|c|}
\hline & \multicolumn{5}{|c|}{$\left(R_{\mathrm{B}}\right)_{\mathrm{UG}}(\mu \mathrm{m})$} & \multicolumn{5}{|c|}{$\left(R_{B}\right)_{\mathrm{SP}}(\mu \mathrm{m})$} & \multicolumn{4}{|c|}{$h / \tan (\beta)(\mu \mathrm{m})$} \\
\hline & \multicolumn{3}{|c|}{ Eq. (23) } & \multirow{2}{*}{$\begin{array}{l}\text { Eq. } \\
\text { (25) }\end{array}$} & \multirow{2}{*}{$\begin{array}{l}\text { Test } \\
\text { data }\end{array}$} & \multicolumn{3}{|c|}{ Eq. (36) } & \multirow{2}{*}{$\begin{array}{l}\text { Eq. } \\
\text { (37) }\end{array}$} & \multirow{2}{*}{$\begin{array}{l}\text { Test } \\
\text { data }\end{array}$} & \multicolumn{3}{|c|}{ Eq. (62) } & \multirow{2}{*}{$\begin{array}{l}\text { Test } \\
\text { data }\end{array}$} \\
\hline & SP & 2D & $\mathrm{CP}$ & & & TB & $2 \mathrm{D}$ & CP & & & SP & $2 \mathrm{D}$ & CP & \\
\hline Fig. 8a & 41.6 & 41.2 & 41.0 & 40.7 & 40.0 & 78.3 & 76.9 & 76.4 & 75.3 & 75.0 & 15.0 & 7.5 & 3.8 & 9.1 \\
\hline Fig. $8 \mathrm{~b}$ & 52.4 & 52.0 & 51.9 & 51.5 & 47.5 & 98.3 & 97.0 & 96.5 & 95.4 & 97.5 & 18.2 & 9.1 & 4.5 & - \\
\hline Fig. 8c & 68.5 & 68.1 & 67.9 & 67.6 & 67.5 & 128.2 & 126.8 & 126.2 & 125.0 & 122.5 & 23.9 & 12.0 & 6.1 & 13.5 \\
\hline
\end{tabular}

To examine the accuracy of Eqs. (25) and (37) further, an extended study on Figs. 7a-c is also carried out by varying the mode I critical ERR of the interface $G_{I c}$ while keeping all other parameters unchanged. Figs. 8a-c shows the variation of $\left(R_{B}\right)_{U G}$ with respect to $G_{I C}$ for the specimens in Figs. 7a-c respectively, using the three mechanical models. The values of $\left(R_{B}\right)_{U G}$ in all the three models converge to the value given by Eq. (25) as $G_{I c}$ decreases. At 
the value $G_{I c}=8.6 \mathrm{~N} / \mathrm{m}$, the predictions of Eq. (23) and Eq. (25) are very close to each other, as also shown in Table 1.

(a) $h=4.9 \mu \mathrm{m}, \sigma_{0}=4.46 \mathrm{GPa}$

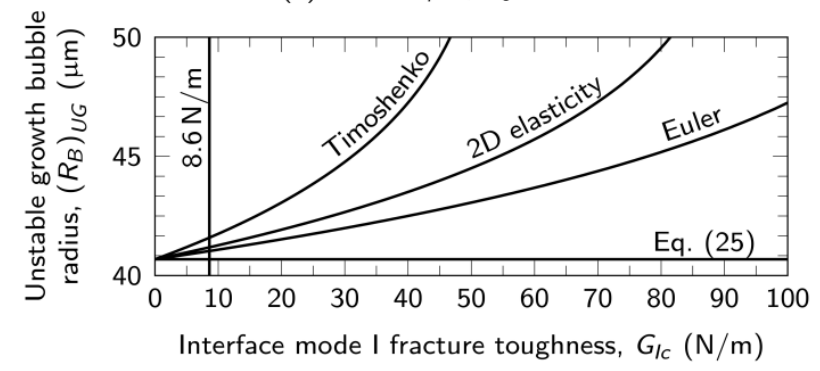

(b) $h=6.2 \mu \mathrm{m}, \sigma_{0}=4.45 \mathrm{GPa}$

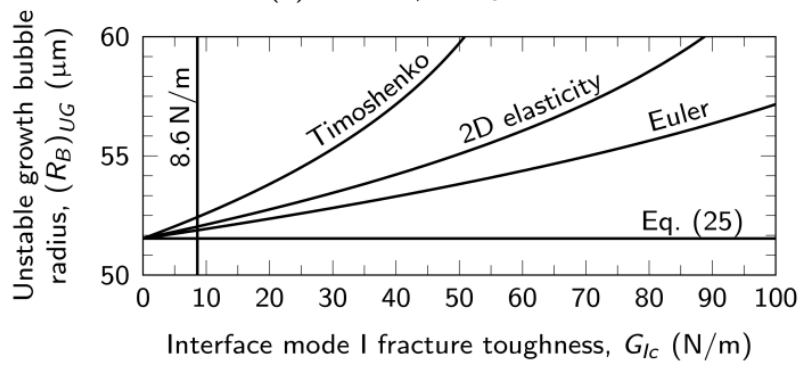

(c) $h=8.0 \mu \mathrm{m}, \sigma_{0}=4.31 \mathrm{GPa}$

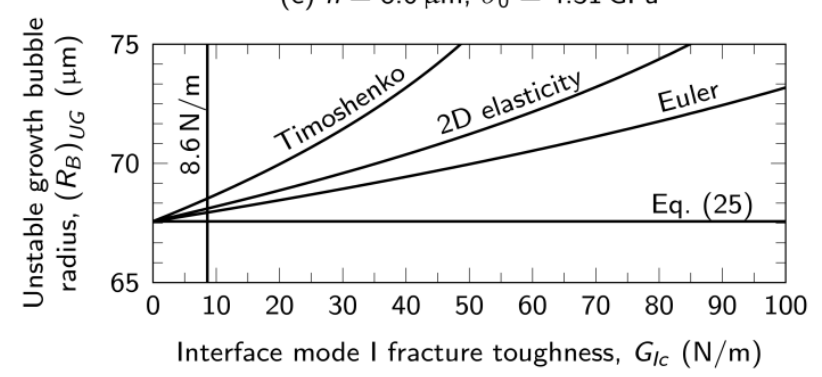

Fig. 9. Variation of the unstable growth bubble radius $\left(R_{B}\right)_{U G}$ with respect to the interface mode I fracture toughness $G_{I c}$ according to (i) Euler beam or classical plate partition theory, (ii) Timoshenko beam or the first-order shear-deformable plate theory, and (iii) 2D elasticity partition theory.

Fig. 10 shows a similar study on the variation of $\left(R_{B}\right)_{S P}$ with respect to $G_{I c}$. Again, the values of $\left(R_{B}\right)_{S P}$ in all the three models converge to the value in Eq. (37) as $G_{I c}$ decreases. At the value $G_{I c}=8.6 \mathrm{~N} / \mathrm{m}$, the predictions of Eq. (36) and Eq. (37) are very close to each other, as also shown in Table 1. Again, the ratio $\psi=G_{\text {IIc }} / G_{\text {Ic }}=5$ is used to calculate the SP and 2D predictions in Figs. 8 and 9. 
(a) $h=4.9 \mu \mathrm{m}, \sigma_{0}=4.46 \mathrm{GPa}$

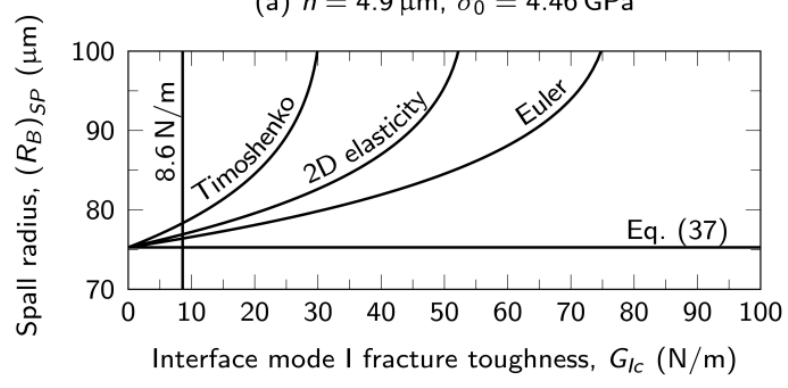

(b) $h=6.2 \mu \mathrm{m}, \sigma_{0}=4.45 \mathrm{GPa}$

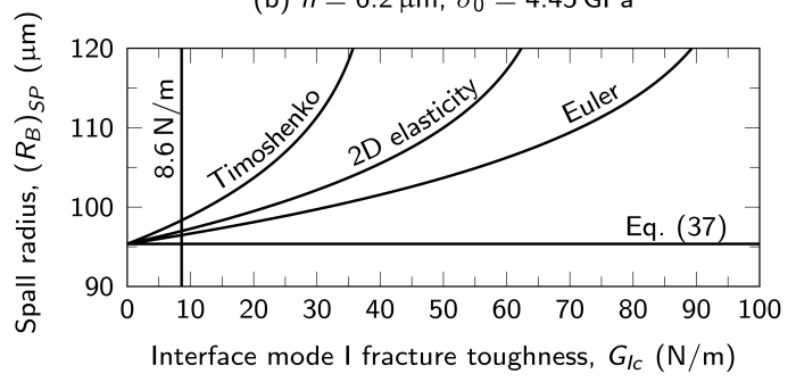

(c) $h=8.0 \mu \mathrm{m}, \sigma_{0}=4.31 \mathrm{GPa}$

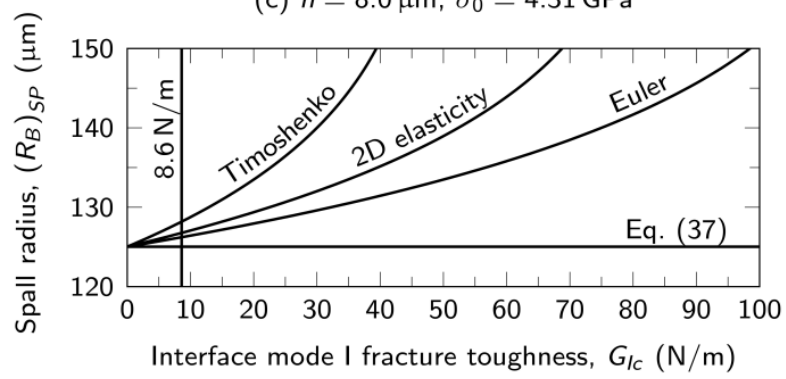

Fig. 10. Variation of the spall radius $\left(R_{B}\right)_{S P}$ with respect to the interface mode I fracture toughness $G_{I c}$ according to (i) Euler beam or classical plate partition theory, (ii) Timoshenko beam or the first-order shear-deformable plate theory, and (iii) 2D elasticity partition theory.

It is desirable to give some explanation for why the three mechanical models give nearly the same predictions for the unstable growth bubble radius and the spall radius. The three mechanical models are all developed based on the assumption of small amplitude-tothickness ratio, that is, $A / h<<1$. One consequence of this assumption is that the crack tip radial force $N_{r B e}$ in Eq. (12) makes no contribution to the total ERR in Eq. (10) and (13). The total ERR is instead solely due to the crack tip bending moment $M_{r B}$ in Eq. (11). This results in a constant fracture mode mixity throughout the whole delamination process. The firstorder shear-deformable plate model predicts a mixed-mode fracture with $G_{I} / G=0.25$; the 2D elasticity model also predicts a mixed-mode fracture with $G_{I} / G=0.6227$; the classical plate model predicts a pure mode I fracture with $G_{I} / G=1.0$. The mode mixity does not have 
much effect on the predictions of the unstable growth bubble radius and the spall radius when $G_{I c}$ is small, that is, all three models give nearly the same predictions, as shown in Figs. 8 and 9 and Table 1.

The mode mixity does, however, affect the amplitude in Eq. (18) of a delamination bubble, the relaxation strain in Eq. (19) and relaxation stress in Eq. (20). These three models therefore give different predictions of these quantities. Due to lack of accurate measurements of these quantities, no comparisons could be made in the present work. Comparison can, however, be made between measurements of the kink-off angle and predictions from Eq. (62). Values of $h / \tan (\beta)$ are also recorded in Table 1 . The critical mode I ERR of the oxide film is $G_{c f}=20 \mathrm{~N} / \mathrm{m}$. For Fig. 8a, $h / \tan (\beta)=9.10 \mu \mathrm{m}$ was measured approximately from Fig. 3 in Ref. [10], which is a similar case. No test value for Fig. 8b was found in Tolpygo and Clarke's studies [9,10]. For Fig. 8c, $h / \tan (\beta)=13.46 \mu \mathrm{m}$ was measured approximately from Fig. $7 \mathrm{f}$ in this work (reprinted from Tolpygo and Clarke's work [10]), which is a similar case. The averages of the measurements of the four diameters at $0^{\circ}, 90^{\circ}$ and $\pm 45^{\circ}$ were used to obtain the test values. It is seen that the $2 \mathrm{D}$ elasticity model gives good predictions but the other two models do not.

It can be concluded at this point that the 2D elasticity model predicts the whole delamination process very well, including the initiation of unstable growth, size of spallation and kink-off angle. The other two models, however, only give good predictions of the initiation of unstable growth and the size of spallation.

As mentioned earlier, Refs. [23-25] show, using data from extensive fracture testing [2631], that the partition theory based on Euler beam or classical plate theory [12-14] gives very accurate predictions of interface fracture toughness for macroscopic mixed-mode fracture while the partition theories based on Timoshenko beam theory or the first-order sheardeformable plate theory [12-14] and 2D elasticity [15-20] give poor predictions. The very latest studies [11,32], however, show that the 2D elasticity partition theory gives accurate predictions for the delamination behavior of micro-scale and nano-scale thin films. This may be expected since the partition theory based on Euler beam or classical plate theory is a 'global' partition theory (that is, cracks develop over finite-size length scales) which governs macroscopic fracture whereas the $2 \mathrm{D}$ elasticity partition theory is a 'local' partition theory (that is, infinitesimal crack growth is assumed) which governs micro- or nano-scale fracture. 
At this point, it is worth noting the following again: All three mechanical models are developed based on the assumption of a small amplitude-to-thickness ratio (i.e. $A / h<<1$ ). For Figs. 7a-c, by using Eq. (26) for the three mechanical models, the ratios at the initiation of unstable growth radii are $(A / h)_{U G T}=0.3470,0.3093$ and $0.2810 ;(A / h)_{U G 2 D}=0.1988$, 0.1772 and 0.1610 ; and $(A / h)_{U G E}=0.1388,0.1237$ and 0.1124 . They are very small for the classical plate model, but are not particularly small for the first-order shear-deformable plate and $2 \mathrm{D}$ elasticity models. Then, the ratios at the respective spall radii are $(A / h)_{S P T}=1.188$, 1.059 and 0.9623; $(A / h)_{S P 2 D}=0.6808,0.6066$ and 0.5513; and $(A / h)_{S P E}=0.4753,0.4235$ and 0.3849. Clearly, these are not particularly small for any of the three models. Since the models predict the spallation behavior very well, they must capture some of key physics of the mechanical process. As mentioned earlier, the direct consequence of the assumption that $A / h<<1$ is that the crack tip radial effective force $N_{r B e}$ in Eq. (12) makes no contribution to the total ERR in Eqs. (10) and (13). Measurements of residual stress in some stationary buckles (see Figs. 8 and 9 in Tolpygo's and Clarke's work [10]) do indeed show that $N_{r B e}=0$ at the crack tip for large values of $A / h$. The total ERR is solely from the crack tip bending moment $M_{r B}$ in Eq. (11). The assumption $A / h<<1$ is therefore no longer required. The only restriction is that the maximum value of $A / h$ is around 1 , which is the usual condition of the von Kármán geometric nonlinearity assumption used in Eq. (5). The above values of $A / h$ are obviously within the limitation.

Finally, more comparisons are performed using samples with oxide films of various thicknesses and residual stresses. The samples had oxide thicknesses in the range $h=0.8-$ $8.0 \mu \mathrm{m}$ (after oxidation for $0.5-100 \mathrm{~h}$ ) and the measured residual compressive stresses were in the range 4-5 GPa. The results are shown in Fig. 11. The solid dots represent measurements on the oxides. The solid line is from Eq. (37). Again, the present mechanical models predict the experimental results very well. Due to absence of experimental results, no comparison is carried out on for spallation with straight edges. 


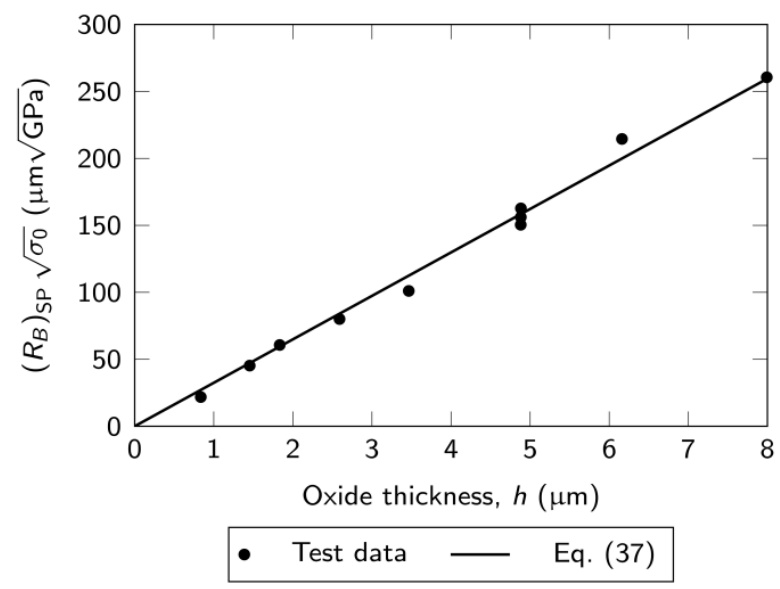

Fig. 11. The spallation parameter $\left(R_{B}\right)_{S P} \sqrt{\sigma_{0}}$ as a function of the oxide thickness with test data from Tolpygo and Clarke’s work [10].

\section{Conclusions}

PECs can be formed by pockets of tensile stress and shear stress on and around the interface between a thin film and a thick substrate, which can be caused by a number of different processes, including thermal effects and chemical effects. PECs can cause the interface spallation failure of thin films. Three mechanical models have been developed to predict several aspects of the spallation failure of elastic brittle thin films by using partition theories for mixed-mode fracture based on classical plate theory, first-order shear-deformable plate theory and full 2D elasticity. Based on experimental results from Tolpygo and Clarke $[9,10]$ for circular-edged delaminations, the three models all give accurate predictions of the initiation of unstable growth of separation bubbles and the size of spallation. The 2D elasticity model also gives accurate predictions of the final kink-off angle but the classical plate and first-order shear-deformable plate models are unable to. The nucleation and stable growth of a separation bubble are solely driven by the bubble energy but unstable growth is driven by both bubble energy and buckling. Final kinking off is controlled by the toughness of the interface and the film and the maximum bubble energy. Note that further experiments would be required in order test the mechanical models for delamination with straight edges.

The present mechanical models reveal a new failure mechanism of thin films under compressive residual stress and will be particularly useful to study the spallation failure of thermal barrier coating material systems. 


\section{Acknowledgements}

The authors gratefully acknowledge Professor D. R. Clarke of Harvard University for valuable discussions.

\section{References}

[1] L.B. Freund, S. Suresh, Thin Film Materials: Stress, Defect Formation and Surface Evolution, Cambridge University Press, 2003.

[2] H. Chai, Three-dimensional fracture analysis of thin-film debonding, Int. J. Fract. 46 (1990) 237-256. DOI: 10.1007/BF00037155.

[3] J.W. Hutchinson, M.D. Thouless, E.G. Liniger, Growth and configurational stability of circular, buckling-driven film delaminations, Acta. Metall. Mater. 40 (1992) 295-308. DOI: 10.1016/0956-7151(92)90304-W.

[4] H.M. Jensen, I. Sheinman, Straight-sided, buckling-driven delamination of thin films at high stress levels, Int. J. Fract. 110 (2001) 371-385. DOI: 10.1023/A:1010821918325.

[5] M.W. Moon, H.M. Jensen, J.W. Hutchinson, K.H. Oh, A.G. Evans, The characterization of telephone cord buckling of compressed thin films on substrates, J. Mech. Phys. Solids 50 (2002) 2355-2377. DOI: 10.1016/S0022-5096(02)00034-0.

[6] H.M. Jensen, I. Sheinman, Numerical analysis of buckling-driven delamination, Int. J. Solids Struct. 39 (2002) 3373-3386. DOI: 10.1016/S0020-7683(02)00158-0.

[7] J.W. Hutchinson, M.Y. He, A.G. Evans, The influence of imperfections on the nucleation and propagation of buckling driven delaminations, J. Mech. Phys. Solids 48 (2000) 709-734. DOI: 10.1016/S0022-5096(99)00050-2.

[8] M.Y. He, A.G. Evans, J.W. Hutchinson, Effects of morphology on the decohesion of compressed thin films, Mater. Sci. Eng. A245 (1998) 168-181. DOI: 10.1016/S09215093(97)00848-4.

[9] V.K. Tolpygo, D.R. Clarke, Spalling failure of $\alpha$-alumina films grown by oxidation: I. Dependence on cooling rate and metal thickness, Mater. Sci. Eng. A278(2000) 142150. DOI: 10.1016/S0921-5093(99)00581-X.

[10] V.K. Tolpygo, D.R. Clarke, Spalling failure of $\alpha$-alumina films grown by oxidation. II. Decohesion nucleation and growth, Mater. Sci. Eng. A278 (2000) 151-161. DOI: 10.1016/S0921-5093(99)00582-1.

[11] S. Wang, C.M. Harvey, B. Wang, Room temperature spallation of $\alpha$-alumina films grown by oxidation, Eng. Fract. Mech. Available online 14 March 2017.

[12] C.M. Harvey, Mixed-mode partition theories for one-dimensional fracture. PhD thesis, University of Loughborough, UK; 2012. URL: https://dspace.lboro.ac.uk/2134/10269.

[13] S. Wang, C.M. Harvey, Mixed mode partition theories for one dimensional fracture, Eng. Fract. Mech. 79 (2012) 329-352. DOI: 10.1016/j.engfracmech.2011.11.013.

[14] C.M. Harvey, S. Wang, Mixed-mode partition theories for one-dimensional delamination in laminated composite beams, Eng. Fract. Mech. 96 (2012) 737-759. DOI: 10.1016/j.engfracmech.2012.10.001.

[15] J.W. Hutchinson, Z. Suo, Mixed mode cracking in layered materials. Adv. Appl. Mech. 29 (1991) 63-191. DOI: 10.1016/S0065-2156(08)70164-9. 
[16] C.M. Harvey, J.D. Wood, S. Wang, A. Watson, A novel method for the partition of mixed-mode fractures in 2D elastic laminated unidirectional composite beams, Compos. Struct. 116 (2014) 589-594. DOI: 10.1016/j.compstruct.2014.05.041.

[17] C.M. Harvey, J.D. Wood, S. Wang, Brittle interfacial cracking between two dissimilar elastic layers: Part 1—Analytical development, Compos. Struct. 134 (2015) 10761086. DOI: 10.1016/j.compstruct.2015.06.080.

[18] C.M. Harvey, J.D. Wood, S. Wang, Brittle interfacial cracking between two dissimilar elastic layers: Part 2-Numerical verification, Compos. Struct. 134 (2015) 1087-1094. DOI: 10.1016/j.compstruct.2015.06.079.

[19] S. Wang, C.M. Harvey, L. Guan, Partition of mixed modes in layered isotropic double cantilever beams with non-rigid cohesive interfaces, Eng. Fract. Mech. 111 (2013) 125. DOI: 10.1016/j.engfracmech.2013.09.005.

[20] J.D. Wood, C.M. Harvey, S. Wang, Partition of mixed-mode fractures in 2D elastic orthotropic laminated beams under general loading, Compos. Struct. 149 (2016) 239_ 246. DOI: 10.1016/j.compstruct.2016.04.016.

[21] H.H. Yu, M.Y. He, J.W. Hutchinson, Edge effects in thin film delamination, Acta. Mater. 49 (2001) 93-107. DOI: 10.1016/S1359-6454(00)00293-7.

[22] H.H. Yu, J.W. Hutchinson, Delamination of thin film strips. Thin Solid Films 423 (2003) 54-63. DOI: 10.1016/S0040-6090(02)00973-2.

[23] S. Wang, C.M. Harvey, B. Wang, A. Watson, Post-local buckling-driven delamination in bilayer composite beams, Compos. Struct. 133 (2015) 1058-1066. DOI: 10.1016/j.compstruct.2015.08.012.

[24] C.M. Harvey, S. Wang, Experimental assessment of mixed-mode partition theories, Compos. Struct. 94 (2012) 2057-2067. DOI: 10.1016/j.compstruct.2012.02.007.

[25] C.M. Harvey, M.R. Eplett, S. Wang, Experimental assessment of mixed-mode partition theories for generally laminated composite beams, Compos. Struct. 124 (2015) 10-18. DOI: 10.1016/j.compstruct.2014.12.064.

[26] B.D. Davidson, P.L. Fariello, R.C. Hudson, V. Sundararaman, Accuracy assessment of the singular-field-based mode-mix decomposition procedure for the prediction of delamination. In: Hooper SJ, editor. Composite materials: testing and design (thirteenth volume), ASTM STP 1242. American Society for Testing and Materials, 1997, pp. 109-128. DOI: 10.1520/STP18272S.

[27] B.D. Davidson, S.J. Gharibian, L.T. Yu, Evaluation of energy release rate-based approaches for predicting delamination growth in laminated composites, Int. J. Fract. 105 (2000) 343-365. DOI: 10.1023/A:1007647226760.

[28] S. Hashemi, A.J. Kinloch, J.G. Williams, The analysis of interlaminar fracture in uniaxial fibre-polymer composites, Proc. R. Soc. A 427 (1990) 173-199. DOI: 10.1098/rspa.1990.0007.

[29] A.J. Kinloch, Y. Wang, J.G. Williams, P. Yayla, The mixed-mode delamination of fibre composite materials, Compos. Sci. Technol. 47 (1993) 225-237. DOI: 10.1016/02663538(93)90031-B.

[30] M. Charalambides, A.J. Kinloch, Y. Wang, J.G. Williams, On the analysis of mixedmode failure, Int. J. Fract. 54 (1992) 269-291. DOI: 10.1007/BF00035361.

[31] M. Conroy, B.F. Sørensen, A. Ivankovic, Combined numerical and experimental investigation of mode-mixity in beam like geometries, In: Proceedings of the 37th Annual Meeting of the Adhesion Society, Feburary 2014, San Diego, California, USA. 
[32] J.D. Wood, C.M. Harvey, S. Wang, Adhesion toughness of multilayer graphene membranes, (ready to submit).

[33] J.R. Reeder, An evaluation of mixed-mode delamination failure criteria, NASA Technical Memorandum 104210, Langley Research Center, Hampton (VA), 1992.

[34] S.P. Timoshenko, J.M. Gere, Theory of Elastic Stability, second ed., McGraw-Hill, New York, 1963.

[35] N.A. Fleck, A.C.F. Cocks, J. Lampenscherf, Thermal shock resistance of air plasma sprayed thermal barrier coatings, J. Eur. Ceram. Soc. 34 (2014) 2687-2694.

DOI: 10.1016/j.jeurceramsoc.2014.01.002. 